\title{
Probing the intracluster star-light with Chandra
}

\author{
A. Finoguenov ${ }^{1,2}$, R. P. Kudritzki ${ }^{3}$, and C. Jones ${ }^{2}$ \\ 1 Max-Planck-Institut für extraterrestrische Physik, Giessenbachstraße, 85748 Garching, Germany \\ 2 Smithsonian Astrophysical Observatory, 60 Garden st., MS 3, Cambridge, MA 02138, USA \\ 3 Institute for Astronomy, University of Hawaii, 2680 Woodlawn Drive, Honolulu, Hawaii 96822, USA
}

Received 5 October 2001 / Accepted 20 March 2002

\begin{abstract}
We propose a method to test for the presence of intracluster star-light using X-ray binaries as a trace population. We discuss a particular application of this method to observations of the Virgo cluster.
\end{abstract}

Key words. stars: binaries: close - X-rays: stars - cosmology: observations

\section{Introduction}

The integrated light and the mass-to-light ratio in galaxy clusters plays an important role in several aspects of observational cosmology. Carlberg et al. (1996), for example, use the mean M/L ratio determined for the CNOC cluster sample to derive an estimate of the matter density of the universe, $\Omega_{\mathrm{o}}$. Mass-to-light ratios are important diagnostics of biased/non-biased star-formation in clusters (Bahcall et al. 2000), a key quantity for the explanation of the heavy element enrichment of the ICM (Arnaud et al. 1992; Renzini et al. 1993), and important for describing the statistical properties of cluster surveys (Borgani \& Guzzo 2001). The classical approach to estimate the optical luminosity of the cluster was to sum the light from the individual member galaxies. A detailed review on the luminosity function of galaxies can be found in Binggeli et al. (1988). Recent studies provide more refined integrated light estimates, adding in dwarf galaxies and an estimate for the amount of diffuse stellar light (also called the intergalactic stellar population, IGSP), as was recently done for the Coma cluster (Gregg \& West 1998).

The result of galaxy merging and galaxy harassment as proposed by Moore et al. (1996) will leave stellar debris that accumulates in the cluster potential. Therefore the observation of an intracluster stellar component would place interesting constraints on the history of galaxy collisions in clusters. The efficiency of tidal stripping is controlled by the probability of a galaxy crossing the cluster core and by the duration of the impact. As simulations show, the two effects compensate each other and the tidal radius is similar among clusters and equals $100 h^{-1} \mathrm{kpc}$ (Klypin et al. 1999).

Send offprint requests to: A. Finoguenov, e-mail: alexis@xray.mpe.mpg.de
Observationally, the determination of the level of such diffuse emission is very difficult due to its low optical surface brightness. It should be observed best in nearby clusters, but there it can easily be confused with background and foreground emission. The present sensitivity limit for optical searches for IGSP corresponds to $20 \%$ of the total light of the cluster (Melnick \& Sargent 1977). This value may still be considered uncertain, as values up to $80 \%$ are still cited (e.g. Feldmeier et al. 1998). One of the solutions proposed to solve this problem is the observation of individual bright objects, that represent the light density of the IGSP. The most important sources are supernovae, red giants and planetary nebulae. For the Virgo Cluster, the detection of intra-cluster red giants (Ferguson et al. 1998) and PN (Arnaboldi et al. 1996; Mendez et al. 1997) provided evidence for the existence of an intracluster stellar population. However recently Kudritzki et al. (2000) showed that many of the intra-cluster PN candidates are Lyman-alpha emitting background galaxies, although Freeman et al. (2000) demonstrated that a significant fraction must be real PN belonging to an intracluster stellar population. Mass estimates for this population suggest that it contains roughly $10 \%$ to $20 \%$ of the stellar mass in the Virgo cluster galaxies. Durrell et al. (2002) using independent method based on the red giant stars confirmed this conclusion.

With the advent of high-angular resolution, X-ray astronomy could play a leading role in this seemingly purely optical field, via studies of the intracluster X-ray binaries.

\section{The method}

Chandra observations reveal a numerous population of galactic X-ray binaries (XRB's) in most elliptical galaxies (Sarazin et al. 2000; Finoguenov \& Jones 2001). With 
luminosities of $10^{37-39} \mathrm{ergs} / \mathrm{s}$, these sources are easily detected even at the distance of Virgo cluster galaxies. The total emitted flux is proportional to the optical luminosity of stars (Matsushita 1998) and within individual galaxies, the number density of the XRB's follows the distribution of the stellar light (Finoguenov \& Jones 2002; Sarazin et al. 2001). Finding one XRB with a luminosity ${ }^{1}$ exceeding $10^{37} \mathrm{ergs} / \mathrm{s}$ corresponds to revealing $4.3 \times 10^{8} L_{\odot}$ light in the $B$ band. Although, the sensitivity of the PN method is higher, $0.5 \times 10^{8} L_{\odot}$ for each detected $\mathrm{PN}$, for bright [OIII] PN the limit $5 \times 10^{8} L_{\odot}$ is used (Durrell et al. 2002). Moreover, the first results for the Virgo PN survey indicate that it is limited by contamination by background objects, rather than the lack of detections. Therefore, we propose to use X-ray binaries, as another indicator for the level of the intracluster stellar population. Given the large field of view of contemporary X-ray telescopes, finding of $1 \mathrm{XRB}$ in a single pointing corresponds to probing the average surface brightness of intracluster star-light on the $\mu_{B}=30 \mathrm{mag} \operatorname{arcsec}^{2}$ level. Typical surface brightness of the intracluster star-light is $\mu_{B} \sim 27 \mathrm{mag} \operatorname{arcsec}^{2}$.

At the moment, only a few Chandra studies of the $\mathrm{X}$-ray binary population in early-type galaxies have been published. Relations between the optical and hard X-ray luminosity, based on ASCA studies alone have a scatter of a factor of 1.5 (Matsushita 2001) for most of the galaxies, while higher X-ray luminosities are met, they are attributed to AGN activity, in the form of bright central point source or hard diffuse X-ray emission. White (2001) demonstrated that the deviation from the $L_{\mathrm{X}}-L_{B}$ relation correlates with the specific frequency of globular clusters. This dependency could easily be eliminated by comparison with the optical image. Our normalization corresponds to the case of nearly $(90 \%)$ clean sample of non-globular XRB.

Theoretical modeling of the XRB's luminosity function predicts its gradual evolution with time since starformation ( $\mathrm{Wu} 2001$ ). Most drastic changes are expected between the disk and the bulge population of XRB's, as revealed by observation of M 81 (Tennant et al. 2001). Therefore, having determined the luminosity function of Virgo intracluster XRB's, we will be able to differentiate between the scenarios for diffuse light production, which is not possible in any other methods.

The feasibility of the XRB detection in Virgo is illustrated in Fig. 1. We assumed that the distribution of diffuse light has a core radius equal to the cluster tidal radius and a total amount of light in the IGSP equal to

\footnotetext{
${ }^{1}$ Spectral characteristics of XRB's are not uniform. Thus to compare different measurements, one should specify the spectral model assumed. We use the disk blackbody model of Makishima et al. (1986) with the characteristic temperature of $0.5 \mathrm{keV}$ and cite the bolometric luminosity. This spectral model corresponds to the soft excess in the XRB, which is well matched to the energy window of Chandra. If we use the mean spectral index of 0.4 instead, the luminosity in the $0.4-10 \mathrm{keV}$ is twice as high. Spectrally harder sources are detected with Chandra at lower countrate.
}

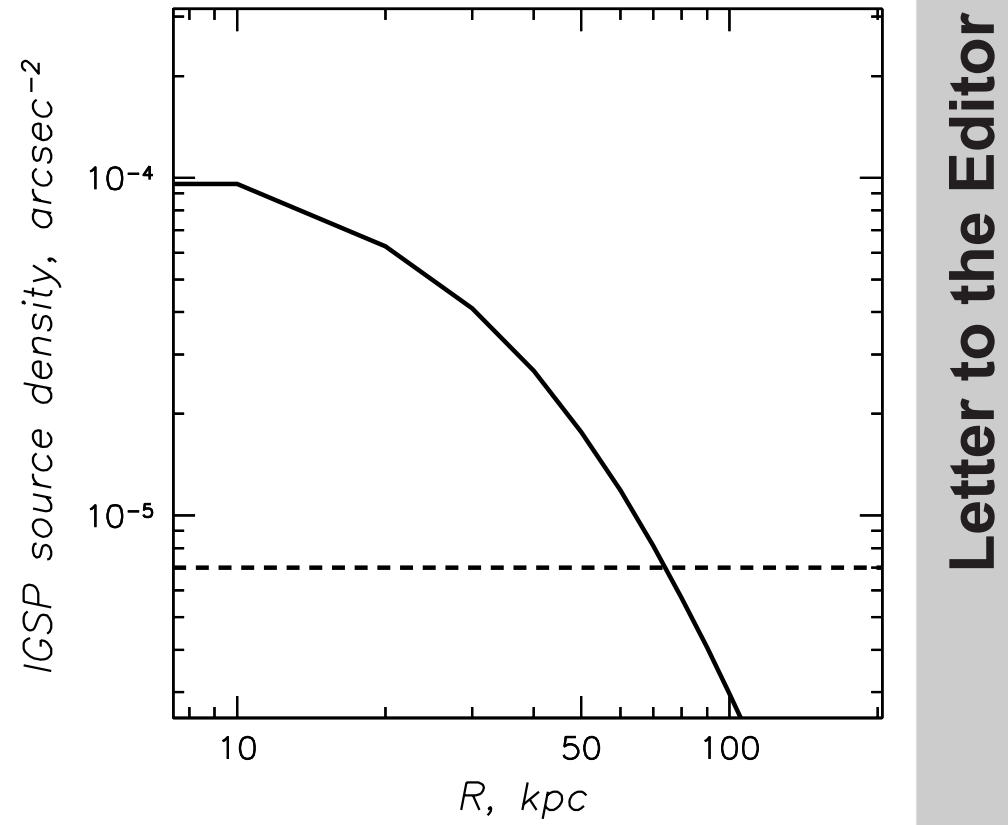

Fig. 1. Predicted X-ray source (for luminosities exceeding $10^{37} \mathrm{ergs} / \mathrm{s}$ ) density of the Intracluster Stellar Population, having $20 \%$ the luminosity of Virgo cluster galaxies (solid line). The dashed line represents $10 \%$ of the expected contribution from CXB from Giacconi et al. (2001). The profile is centered on the concentration of early-type galaxies between M 86 and M 87, as found by Schindler et al. (1999).

$20 \%$ of the total light in Virgo galaxies, according to the revised estimate using observations of planetary nebulae (Freeman et al. 2000).

Using the model presented in Fig. 1, detection of $1 \mathrm{XRB}$ (with luminosity exceeding $10^{37} \mathrm{ergs} / \mathrm{s}$ ) corresponds to an IGSP contribution of $0.2 \%$ to the total light in the Virgo cluster. The gradient in the source number density is expected to be sharp (see Fig. 1). The amplitude of the CXB correlation function on such angular scales $\left(10^{\prime}\right)$ is also $10 \%$ (Vikhlinin \& Forman 1995; Giacconi et al. 2001). This is therefore the principal limitation of the method, unless optical follow-up or X-ray spectral information is used. The resulting sensitivity is $\sim 1 \%$ of the total light in the Virgo cluster. Emission from the hot X-ray gas in the Virgo cluster, acting as a background, puts stringent requirements on the angular resolution. For regions in the Virgo cluster close to M 87 (less than half a degree), even for Chandra, variation of the PSF (point spread function) over the field of view (Weisskopf et al. 1996) results in the strongly decreasing sensitivity.

Study of the spectral characteristics of the XRB in M 84 have revealed that they are spectrally different from the constituents of the CXB (Finoguenov \& Jones 2002). Of particular interest are the binaries with a soft component, which exhibit very similar spectra, characterized by a multicolor disk black-body model (Makishima et al. 1986; diskbb model in XSPEC) with a central blackbody temperature of $0.5 \mathrm{keV}$. For the luminosity range of interest $\left(10^{37}-10^{39} \mathrm{ergs} / \mathrm{s}\right)$, over $50 \%$ of the XRB in M 84 
exhibit such a spectrum. By limiting the sources to only those, we can reduce substantially the contamination by CXB to the sample and thus increase the sensitivity of this method to the limit implied by the quality of the hardness ratio determination (typically 30 source counts are required). For an ACIS-I exposure of $40 \mathrm{ksec}$ (or ACIS-S exposure of $25 \mathrm{ksec}$ ), such determination will be possible for one third of the sample, with a resulting sensitivity is $1 \%$ of the total light in the Virgo cluster. Most of the background objects will be type-1 QSO, for which optical counterpart can readily be found in USNO A2.0 catalogs. Type-2 QSO do not overlap in X-ray colors with XRBs due to characteristic strong absorption. This allows for an independent check of the X-ray color selection.

Detection of PNs was also reported for the field far off the Virgo center. Due to lower flux from the thermal gas the requirements on the quality of imaging are reduced, so routine detection of XRBs in fore-coming XMM shallow surveys, (covering large areas, typical for X-ray surveys, challenging for optics) will be feasible.

The proposed X-ray method for estimating the amount of intracluster star-light provides a useful alternative to optical methods and is less affected by systematics in the search of candidates, which was recently realized to be a problem for the PN (Planetary Nebulae) method (Kudritzki et al. 2000). Therefore the use of X-ray observations provide an important test of the results obtained optically. In addition, the shape of the luminosity function of the X-ray binaries can shed light on the age of the intracluster stellar population.

Acknowledgements. The authors thank the referee, Jimmy Irwin, for useful suggestions on the manuscript. This work was supported by NASA grants GO0-1045X and AG5-3064 and the Smithsonian Institution. AF has benefited from discussions with Hans Boehringer. AF acknowledges receiving the Max-Plank-Gesellschaft Fellowship.

\section{References}

Arnaboldi, M., Freeman, K. C., Mendez, R. H., et al. 1996, ApJ, 472, 145

Arnaud, M., Rothenflug, R., Boulade, O., Vigroux, L., \& Vangioni-Flam, E. 1992, A\&A, 254, 49
Bahcall, N. A., Cen, R., Davé, R., Ostriker, J. P., \& Yu, Q. 2000, ApJ, 541, 1

Binggeli, B., Sandage, A., \& Tammann, G. A. 1988, ARA\&A, 26,509

Borgani, S., \& Guzzo, L. 2001, Nature, 409, 39

Carlberg, R. G., Yee, H. K. C., Ellingson, E., et al. 1996, ApJ, 462, 32

Durrell, P. R., Ciardullo, R., Feldmeier, J. J., Jacoby, G. I., \& Sigurdsson, S. 2002, ApJ, in press [astro-ph/0201219]

Feldmeier, J. J., Ciarullo, R., \& Jacoby, G. H. 1998, ApJ, 503, 109

Ferguson, H. C., Tanvir, N. R., \& von Hippel, T. 1998, Nature, 391,461

Finoguenov, A., \& Jones, C. 2001, ApJ, 547, 107

Finoguenov, A., \& Jones, C. 2002, ApJ, submitted

Freeman, K. C., Arnaboldi, M., Capaccioli, M., et al. 2000, ASP Conf. Proc., 197, 389

Giacconi, R., Rosati, P., Tozzi, P., et al. 2001, ApJ, 551, 624

Gregg, M. D., \& West, M. J. 1998, Nature, 396, 359

Klypin, A., Gottloeber S., Kravtsov, A., \& Khokhlov, A. 1999, ApJ, 516, 530

Kudritzki, R. P., Méndez, R. H., Feldmeier, J. J., et al. 2000, ApJ, 536, 19

Makishima, K., Maejima, Y., Mitsuda, K., et al. 1986, ApJ, 308,635

Matsushita, K. 1998, Ph.D. Thesis, The University of Tokyo

Melnick, K., \& Sargent, W. L. W. 1977, ApJ, 215, 401

Mendez, R. H., Guerrero, M. A., Freeman, K. C., et al. 1997, ApJ, 491, L23

Moore, B., Katz, N., Lake, G., Dressler, A., \& Oemler, A. Jr. 1996, Nature, 379, 613

Renzini, A., Ciotti, L., D'Ercole, A., \& Pellegrini, S. 1993, ApJ, 419, 52

Sarazin, C. L., Irwin, J. A., \& Bregman, J. N. 2000, ApJ, 544, L101

Sarazin, C. L., Irwin, J. A., \& Bregman, J. N. 2001, ApJ, 556, 533

Schindler, S., Binggeli, B., \& Böhringer, H. 1999, A\&A, 343, 420

Tennant, A. F., Wu, K., Ghosh, K. K., Kolodziejczak, J. J., \& Swartz, D. A. 2001, ApJ, 549, L43

Vikhlinin, A., \& Forman, W. 1995, ApJ, 455, L109

Weisskopf, M. C., O'dell, S. L., \& van Speybroeck, L. P. 1996, SPIE, 2805, 2

White, R. E. III 2001, preprint [astro-ph/0111293] 